\title{
EDUCACIÓN, CIENCIA Y TECNOLOGÍA \\ LA OPORTUNIDAD DEL DESARROLLO SEGURO LOGístico DE LA FuERzA AÉrEA
}

CT. MARCOS MANUEL GUZMÁN

Jefe Departamento de Investigación - IMA

La oportunidad del desarrollo en ciencia y tecnología en la Fuerza Aérea está intrínsicamente ligada al desarrollo de la educación, como lo demuestra el estudio "Colombia, al filo de la oportunidad", el resultado de 10 meses de investigaciones y estudio del estado real y actual de la educación, ciencia y tecnología en Colombia. Fue realizado por la Misión de Ciencia, Educación y Desarrollo: 10 personas comisionadas por el Gobierno Colombiano con el fin de "buscar nuevos derroteros para orientar a Colombia en su entrada al tercer milenio." Como dijo el Presidente al instalar la misión:

\section{"El árbol del desarrollo social y económico solo puede arraigar y crecer frondoso, si tiene como fundamento el desarrollo tecnológico y el mejoramiento de la calidad de vida de nuestros compatriotas. Estos, a su vez, no lograrán este objetivo sin contar con la ciencia y la tecnología, y sin una reorientación de la educación, para que en ella primen el dominio de la ciencia y el estimulo a la creatividad."}

El documento destaca vez tras vez la estrecha conexión entre educación, ciencia y tecnología, y desarrollo. En el mundo de hoy, el desarrollo se hace con base en la ciencia y tecnología, y éstos, a su vez, dependen de la educación. Hablando de algunos países en vías de desarrollo, el estudio dice: "Estos países impulsaron su desarrollo con un plan concertado a largo plazo, comprometiendo estratégicamente los sistemas políticos y económicos con la inversión en el sistema educativo y en el de ciencia y tecnología, así como con su reconceptualización, para poder alcanzar niveles primer mundistas en menos de 25 años." Refiriéndose ya a la situación específica de Colombia, el texto habla de estudios que "han demostrado la clara relación que existe entre un capital humano mejor calificado y el crecimiento económico, así como con el mejoramiento de las condiciones del mercado laboral. (...) La liberalización, internacionalización y modernización de la economía exige una población educada en escuela primaria, secundaria y superior, con mejores ingresos, mayores ahorros, un crecimiento demográfico reducido, inflación controlada, y con más técnicos y científicos de alto nivel que promuevan el desarrollo y la innovación productiva." Hablando de lo que se busca para Colombia en los próximos 25 años, el texto dice: "Un flujo continuo pasará entre la educación y la ciencia y la tecnología. (...) Estos se relacionarán directamente al crecimiento económico y al desarrollo social del país." 
Entonces, ¿cómo está la Fuerza Aérea en el tema de la educación? Conscientes de la necesidad de incrementar la calidad de la educación para implementar el desarrollo de la ciencia y la tecnología en la Fuerza Aérea, se vislumbran claramente unas políticas establecidas por el alto mando mediante un planeamiento estratégico institucional para mejorar la calidad en las instituciones educativas aéreas, con la profesionalización de sus oficiales y sub-oficiales al otorgar los títulos de Administrador Aeronáutico, Ingenieros Mecánicos y de Sistemas, Tecnólogos en Mantenimiento Aeronáutico, en Comunicaciones, en Electrónica,

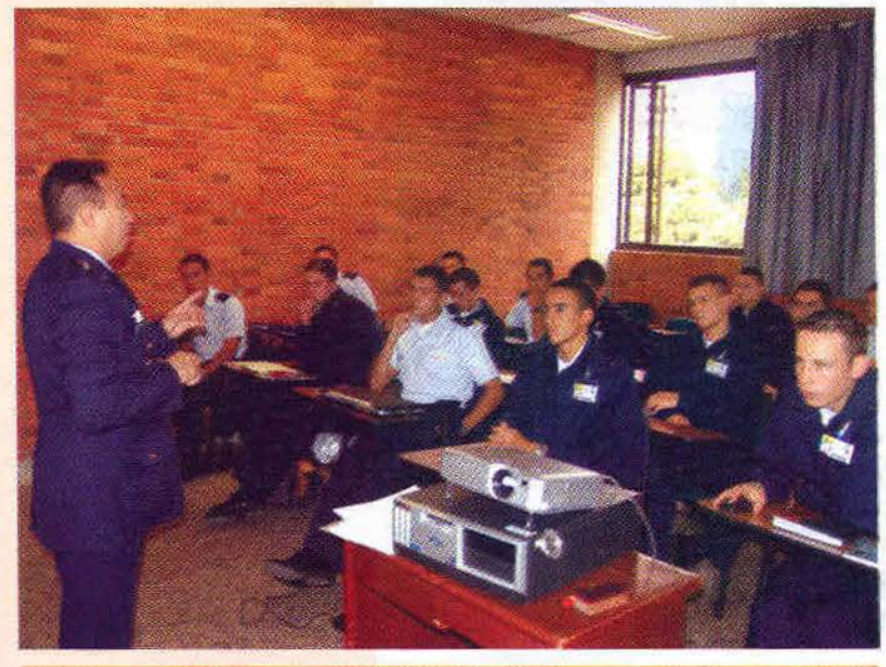
en Seguridad y Defensa de Bases. Del mismo modo, ha desarrollado programas de postgrado y diplomados acordes con las necesidades propias de la institución, como Especialización en Gerencia de la Seguridad Aérea, en Logística Aeronáutica, y próximamente la Especialización en Operaciones Aéreas, diplomados en Prevención de Accidentes, Gerencia del Recurso Humano, Docencia Universitaria, y otros postgrados y diplomados que cumplan con las necesidades estratégicas institucionales y optimicen el cumplimiento de la misión. También se está buscando obtener la certificación de procesos, el registro calificado de los programas de postgrados en el IMA y la acreditación de los programas de las escuelas de formación, de acuerdo con los últimos lineamientos del Ministerio de Educación a través de CONACES y el Consejo Nacional de Acreditación.

Por otro lado, la Fuerza Aérea está haciendo énfasis operativo en el desarrollo de la investigación en el entorno de la institución aérea, bajo los lineamientos de ahorro institucional, generación de conocimiento aeronáutico y disminución de dependencia tecnológica. Para motivar e incentivar a la investigación, el comando de la Fuerza Aérea le destinó el 3\% del presupuesto de los fondos internos, se creó el Centro de investigación y tecnología aeronáutica (CITA), se diseñó el Premio Anual de Ciencia y Tecnología FAC, el cual fue otorgado en su primera versión al Comando Aéreo de Combate No. 5 en el año 2.004 y en el año 2005 al Comando Aéreo de Combate No. 4. También se crearon las secciones de Investigación y Desarrollo Tecnológico en cada unidad de la FAC, y se están registrando las patentes y derechos de autor de los proyectos e investigaciones realizados por miembros de la Fuerza Aérea, como también la inscripción de grupos de investigación en Colciencias por parte del IMA.

Al respecto de lo anteriormente dicho, todavía hay mucho terreno para caminar para llegar a la meta fijada por los miembros de la Misión de Ciencia y Tecnología. Qué bueno que seamos nosotros los miembros del Instituto Militar Aeronáutico quienes asumamos el reto del desarrollo en educación, ciencia y tecnología y engrandezcamos aun más nuestra Fuerza Aérea Colombiana y siendo gestores y contribuyentes de un camino más seguro para nuestro país.

\section{BIBLIOGRAFÍA}

"XX Congreso Nacional de Física," Semilla Universitaria \#22, Quindío, Agosto 2.003

Misión de Ciencia, Educación y Desarrollo. Colombia: Al filo de la oportunidad. Informe conjunto. Colección Mesa Redonda, Cooperativa Editorial Magisterio. 